\title{
Geschlechteraspelkte in der Schmerzwahrnehmung
}

Schmerz ist ein äußerst individuelles Symptom - Die Wahrnehmung von Schmerz, seine möglichen Ursachen und Bewältigungsstrategien unterscheiden sich von Mann zu Frau, mit Konsequenzen für die Therapie Miriam Schopper 


\section{Zusammenfassung}

Schmerz ist mehr als ein körperliches Symptom. Er kann Ausdruck von Gefühlen sein wie Kummer oder Einsamkeit, aber auch soziale oder berufliche Belastungen auf körperlicher Ebene spiegeln. Dass Männer und Frauen auf unterschiedliche Weise mit Schmerz umgehen, legt die Empirie seit Langem nahe. Wissenschaftliche Untersuchungen bestätigen dies zunehmend für die Schmerzwahrnehmung und Schmerzunterhaltung, mit Konsequenzen für die Therapie: Ein geschlechterdifferentes Vorgehen empfiehlt sich v.a. in der Pharmakotherapie, den Copingstrategien und der Chronifizierungsprävention.

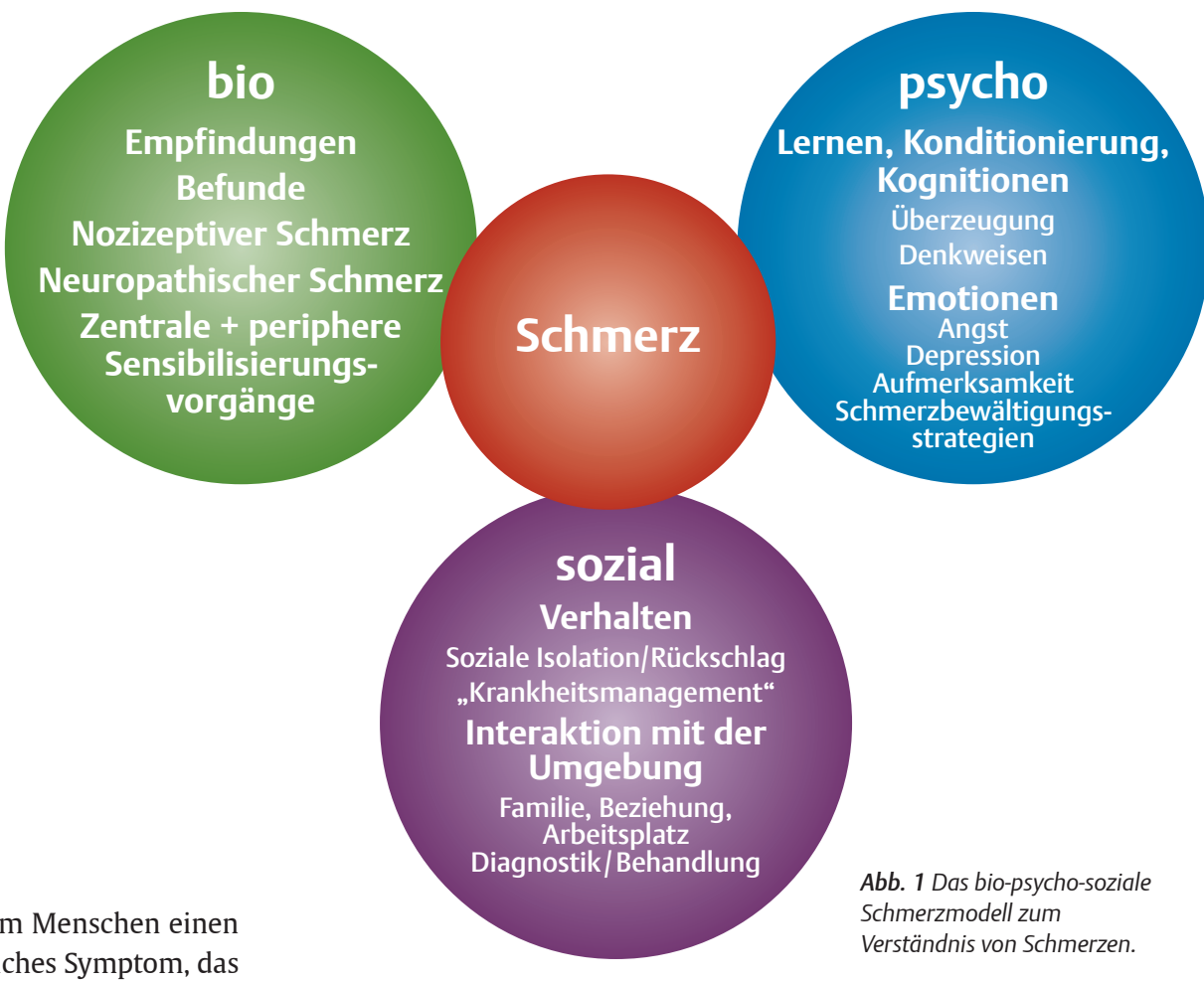

Schmerz ist einer der häufigsten Gründe, warum Menschen einen Arzt aufsuchen. Er ist aber mehr als ein körperliches Symptom, das kuriert werden soll. Je nach Individuum und Situation kann er Mittel zum Ausdruck von Gefühlen, Aufforderung zur Kommunikation oder Instrument zum Erreichen eines Zieles sein. Nicht immer ist dem betroffenen Menschen - oder seinem Arzt - die Vielschichtigkeit seiner Beschwerde bewusst.

Dies ist umso erstaunlicher, da wir alle, Männer und Frauen, Kinder und Alte, Ärzte und Patienten, dieses Phänomen gut kennen. Körperlich und auch seelisch schmerzhafte Erfahrungen sind regelmäßige und normale Ereignisse in unserem Leben. Gerade weil Schmerzen ein allgemein bekanntes, aber immer subjektives und gleichzeitig komplexes Erlebnis darstellen, werden sie unterschiedlich bewertet und unterliegen starken soziokulturellen Einflüssen in der individuellen Entwicklung: „Ein Indianer kennt keinen Schmerz“. Postuliert man, dass der Indianer in unseren Breiten meist ein kleiner Junge ist, stellt sich schon hier die Frage, ob Schmerzen abhängig vom Geschlecht verschieden empfunden werden (sollen).

Unabhängig von der unterschiedlichen Wahrnehmung können wir alle ohne Schmerzempfindung und -verarbeitung aber auch nicht überleben. Die Folge einer kompletten Unempfindlichkeit gegen Schmerzen wären andauernde Verletzungen, die unsere Lebenserwartung deutlich reduzieren würden.

\section{Akute und chronische Schmerzen}

Akute Schmerzen erfüllen eine physiologische Warnfunktion, die uns bei bzw. vor weiterer Gefahr für unseren Körper in Alarmbereitschaft versetzen. Sie werden durch psychosoziale Faktoren wie Angst beeinflusst, aber selten von ihnen allein ausgelöst oder unterhalten.

Chronische Schmerzen hingegen können weiter bestehen, obwohl die körperlichen Ursachen ausgeheilt sind, oder entstehen ohne somatische Ursache auf dem Boden von Ängsten, Depressionen oder anderweitig schwierigen Lebensumständen. In jedem Fall sind sie wesentlich von psychischen und sozialen Umständen beeinflusst. Schmerzen sind also immer gleichzeitig eine emotionale und eine körperliche Erfahrung.

Die Definition von Schmerzen berücksichtigt diese Tatsache explizit:

Schmerz ist ein unangenehmes Sinnes- und Gefühlserlebnis, das mit aktuellen oder potenziellen Gewebeschädigungen verknüpft ist oder mit Begriffen solcher Schädigungen umschrieben wird [1].

Körper und Seele lassen sich nie, weder bei Gesundheit noch bei Krankheit, definitiv trennen, da psychische Prozesse biologische Effekte auslösen und umgekehrt. Deshalb wird heute zum Verständnis von Schmerzen das sog. bio-psycho-soziale Schmerzmodell herangezogen. Zusätzlich zu körperlichen und seelischen Faktoren tragen gesellschaftliche oder kulturelle Einflüsse, Umweltbedingungen sowie familiäre oder berufliche Konstellationen wesentlich zu unserem Wohlbefinden oder zu pathologischen Zuständen bei (Abb.1).

Zieht man die vielfältigen Einflüsse in Betracht, die bei der Schmerzentstehung wirksam werden, ist es nicht verwunderlich, dass sich zumindest in der alltäglichen Wahrnehmung Frauen und Männer in Bezug auf Schmerzen stark unterscheiden. In den letzten 2 Jahrzehnten hat sich auch die Forschung ausführlich mit geschlechtsspezifischen Unterschieden bei Schmerzen beschäftigt.

\section{Wissenschaftliche Erkenntnisse Frauen vulnerabler als Männer}

Chronische Schmerzen treten nach aktuellem Kenntnisstand häufiger bei Frauen auf [2,3]. Bei akuten Schmerzen stehen noch zu we- 


\section{Gender und Gendermedizin}

Der Begriff Gender bezieht sich nicht auf das biologische Geschlecht (englisch: Sex), also X und $Y$ Chromosomen, sondern auf die Rollen, Verhaltensweisen, Aktivitäten und Attribute, die in einer bestimmten Gesellschaft für Männer oder Frauen biologischen als auch mit den psychologisch und sozial bedingten Ursachen für Unterschiede bei Symptomen, Ausprägungen und Auslösern von Krankheiten bei Frauen und Männern.

WHO-Definition

Gender refers to the socially constructed roles, behaviours, activities and attributes that a given society considers appropriate for men and women.

Quelle: www.who.int als adäquat gelten.

Die Gendermedizin beschäftigt sich sowohl mit den

spiegeln. Am Ende einer Schwangerschaft steigen jedoch die Schmerzschwellen unter dem Einfluss hoher Östrogenspiegel. Die Wirkung von Östrogenen auf die Schmerzempfindung scheint nicht nur von der absoluten Höhe des Hormonspiegels, sondern auch vom zeitlichen Verlauf abhängig zu sein. Für Androgene konnten klinisch bisher v. a. antinozizeptive Effekte gezeigt werden [7]. Da die Wirkung der Steroidhormone von Alter, weiblichem Zyklus oder der Anwendung von Kontrazeptiva und Hormonersatztherapien beeinflusst wird, sind entsprechende Untersuchungen bei Frauen und Männern methodisch sehr aufwändig und die Ergebnisse insgesamt bis heute nicht einheitlich.

\section{Schmerzschwellen und -toleranzen}

Großes Interesse galt auch der Untersuchung von Schmerzschwellen und -toleranzen. Insgesamt wird nach heutigem Kenntnisstand davon ausgegangen, dass Frauen bei den meisten getesteten Schmerzqualitäten (z.B. Druck oder thermische Reize) empfindlicher als Männer reagieren [8]. Allerdings wurden diese Studien meist an gesunden Probanden und nicht an Schmerzkranken durchgeführt. Da die Methodik nicht immer einheitlich war (Alter der Probanden, Versuchsaufbau, sozialer und kultureller Hintergrund u.a.) und die meisten Studien keine Aussagen zum Zyklusstag der untersuchten Probandinnen machen, sind die Ergebnisse noch nicht als endgültig zu werten.

nige Daten für eine abschließende Bewertung zur Verfügung. Es gibt jedoch Hinweise, dass Frauen insbesondere mit Hinblick auf die Schmerzstärke sensibler sind [4]. Als gesichert gilt, dass das weibliche Geschlecht ein Risikofaktor für die Chronifizierung von Rückenschmerzen und postoperativen Schmerzen ist [5, 6]. Frauen scheinen also insgesamt vulnerabler als Männer zu sein.

Die Ursachen sind gemäß dem bio-psycho-sozialen Schmerzmodell in verschiedenen Bereichen zu suchen.

Auf genetischer Ebene konnten einige sog. Einzelnukleotidpolymorphismen (Single Nucleotide Polymorphism, SNP), also Variationen einzelner Basenpaare in einem DNA-Strang, identifiziert werden, die sich bei Männern und Frauen unterschiedlich auf die Schmerzwahrnehmung auswirken [6].

Auch die Gehirne von Männern und Frauen unterscheiden sich hinsichtlich ihrer Struktur und ihrer Funktion. Allerdings steht die Forschung diesbezüglich noch am Anfang.

\section{Hormonelle Einflüsse}

Naheliegend ist, dass die Sexualhormone hier Einfluss nehmen. Sie werden nicht nur in den Gonaden, sondern an vielen Orten im Körper, z. B. eben auch im Gehirn, synthetisiert. Östrogen, Progesteron und Testosteron modulieren bei beiden Geschlechtern nicht nur die Entwicklung, sondern auch die Funktion von Organen und Körpersystemen. Stark zusammengefasst wirken Östrogene eher exzitatorisch im Nervensystem und proinflammatorisch auf das Immunsystem, während Progesteron in beiden Systemen eher gegenteilige Effekte zeigt. Testosteron hat tendenziell eine immunsupprimierende Wirkung. Im zentralen Nervensystem entfaltet es seine Wirkung v.a. über seine Metaboliten. Diese kann anregend oder hemmend sein [7].

\section{Schmerzverschlechterung bei hohen Östrogenspiegeln}

Auch Beobachtungen aus der Klinik geben Hinweise auf eine Verschlechterung chronischer Schmerzen, verursacht z. B. durch Migräne oder kraniomandibuläre Dysfunktion, bei hohen Östrogen-

\section{Begleitumstände}

Wie bei experimentellen Versuchsaufbauten, sind auch im normalen Leben Begleitumstände von großer Bedeutung für die Schmerzempfindung. Zum Beispiel gehen psychische Leiden oft mit körperlichen Schmerzen einher. Nicht selten sehen Schmerzspezialisten Patienten, deren Schmerzen ein Hauptsymptom ihrer Depression oder Posttraumatischen Belastungsstörung sind. Von beiden Erkrankungen sind Frauen häufiger als Männer betroffen [9]. In diesen Zusammenhang fällt auch die traurige Realität von körperlichem oder seelischem Missbrauch in Kindheit und Jugend. Eine solche Erfahrung erhöht das Risiko für chronische funktionelle Störungen und Schmerzen im Erwachsenenalter [10]. Mädchen und junge Frauen werden laut Literatur eher Opfer von sexuellem Missbrauch als Männer [11].

\section{Bewältigungsstrategien}

Nicht nur Erlebnisse, positiv oder negativ, entscheiden über unser Befinden. Die Entwicklung von chronischen Schmerzen wird auch maßgeblich durch unsere Strategien im Umgang mit schmerzhaften Erfahrungen beeinflusst. Einstellungen und Verhaltensweisen, Gegebenheiten in der Familie und am Arbeitsplatz können Chronifizierungsvorgänge fördern (s. Kasten S.13).

Als ungünstige Herangehensweise gilt z. B. das „Katastophisieren“. Der Betroffene fühlt sich hilflos und leidet unter einer ständigen gedanklichen Auseinandersetzung mit dem Erlebten und der (unbewussten) Übertreibung des Problems. Diese Copingstrategie scheint eher von jungen Mädchen als von jungen Männern angewendet zu werden [12]. Frauen und Mädchen suchen wohl auch mehr soziale Interaktion, um mit Belastungen umzugehen als Männer oder Jungen, die sich eher ablenken [12].

Diskutiert wird auch, ob die Geschlechtsrollenorientierung oder auch das Lernen am Modell, z. B. bei einer schmerzkranken Mutter, 


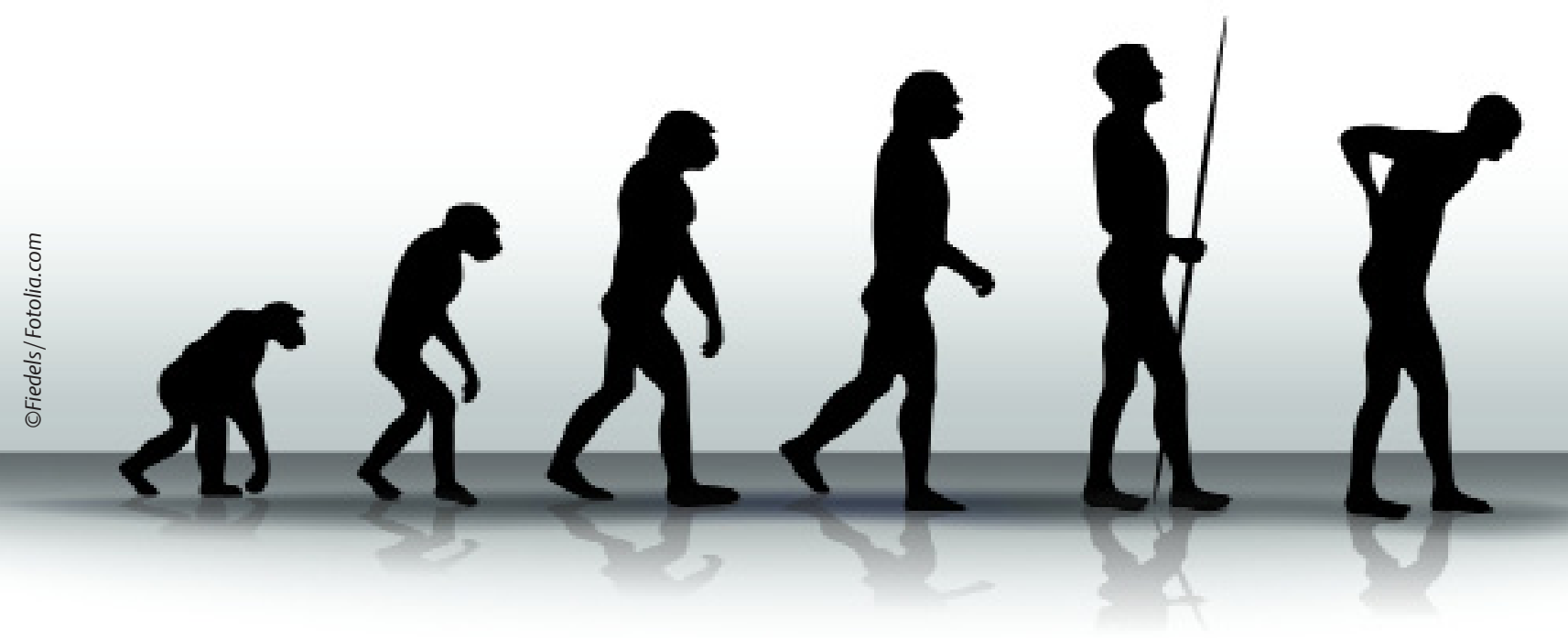

\section{Risikofaktoren für eine Chronifi- zierung von Rückenschmerzen}

das eigene Risiko für chronische Schmerzen erhöht. Eine Untersuchung zur somatoformen Schmerzstörung kam hier zu folgendem Ergebnis: Erkrankte beider Geschlechter neigten bei der Selbstbeschreibung in signifikant größerem Maße zu femininen Rollennormen als gesunde Kontrollpersonen. Diese fanden sich eher in traditionell maskulinen Rollennormen und Eigenschaften wieder [13]. Insgesamt stellen sich bei den meisten dieser Verhaltensweisen und Phänomene noch viele interessante Fragen bez. geschlechtsspezifischer Unterschiede.

\section{Was die Empirie schon seit Langem}

\section{nahelegt, bestätigen nun Ergebnisse der Geschlechterforschung - Frauen nehmen} Schmerzen anders wahr als Männer.

\section{Konsequenzen für die Therapie Arzneimitteltherapie}

Spannend ist jetzt schon die Frage nach der therapeutischen Konsequenz. Hier rücken zunehmend Medikamente in den Fokus. In Deutschland wird leider erst seit 2004 vom Arzneimittelgesetz gefordert, „dass die vorgelegten Unterlagen zur klinischen Prüfung auch geeignet sein müssen, den Nachweis der Unbedenklichkeit oder Wirksamkeit eines Arzneimittels einschließlich einer unterschiedlichen Wirkungsweise bei Frauen und Männern zu erbringen“ [14].

Die meisten Substanzen, die in der Schmerztherapie verwendet werden, wurden schon vor dieser Bestimmung entwickelt und zugelassen, sodass nur wenige Daten auf diesem Gebiet vorliegen. Gesichert ist aber, dass es erhebliche Unterschiede in der Pharmakokinetik, d. h. bei Resorption, Verteilung, Metabolisierung und Ausscheidung, gibt. Eine Hauptrolle spielt hier das Cytochrom-p450-System in der Leber, das für die Verstoffwechslung der meisten Medikamente zuständig ist. Die einzelnen Subtypen dieser großen Enzymfamilie unterscheiden sich in ihrer Aktivität nicht nur zwischen den Geschlechtern, sondern auch zwischen unterschiedlichen Ethnien $[7,15]$. Auch die Wirkung eines Medikaments am Rezeptor (Pharmakodynamik) kann bei Männern und Frauen möglicherweise unterschiedlich stark sein [16]. Solche Effekte konnten an Opioidrezep- (modifiziert nach [31, 32])

Verhalten

- ausgeprägtes Schonverhalten

- Rückzug von normalen Alltagsaktivitäten

- ausgeprägtes Vermeidungsverhalten

- extreme Schmerzangaben

- Medikamentenmissbrauch

Kognitionen

- Katastrophisieren

- Bagatellisieren

- geringe Selbstwirksamkeitserwartung

Arbeitsplatzfaktoren

- Überzeugung, dass die Arbeitstätigkeit dem Körper schadet

- wenig unterstützende Umgebung am

Arbeitsplatz

- kein Interesse von Vorgesetzten oder Kollegen

- Unzufriedenheit am Arbeitsplatz

- fehlender finanzieller Anreiz, Arbeit wieder aufzunehmen

- Rentenwunsch

- geringe Bildung, geringer sozioökonomischer Status

latrogene Faktoren

- Unterstützung von Schonverhalten

- Inanspruchnahmeverhalten

- mehrere (z. T. sich widersprechende) Diagnosen

- katastrophisierende Äußerungen des Arztes

Soziale Faktoren

- gravierende partnerschaftliche/familiäre Konflikte

- überprotektiver Partner

Emotionen

- depressive Stimmungslage

- hohe Aufmerksamkeit/Ängstlichkeit bez. des eigenen Körpers 


\section{Ein geschlechterdifferentes therapeutisches}

\section{Vorgehen in der Praxis empfiehlt sich v.a. für Copingstrategien, Pharmakotherapie und \\ Chronifizierungsprävention.

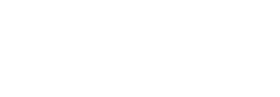

toren bereits gezeigt werden [16, 17]. Leider ist das vorhandene Wissen im Moment noch nicht ausreichend, um geschlechtsspezifische Dosierungsempfehlungen geben zu können.

Merke: Eine ganz wesentliche Tatsache aber ist gut belegt: Frauen sind generell gefährdeter, unter unerwünschten Arzneimittelwirkungen zu leiden [18].

Methoden der Komplementärmedizin werden wohl unterschiedlich häufig angewandt. Laut einer deutschen Untersuchung nutzen Frauen Akupunktur, Homöopathie, Osteopathie und Phytotherapeutika öfter als Männer. Frauen scheinen bei chronischen Schmerzen auch besser von Akupunktur zu profitieren als Männer [7, 19].

\section{Multimodale Therapiestrategien}

Zur Behandlung chronischer Schmerzzustände ist meist keines der genannten Therapieverfahren allein ausreichend. Angezeigt ist eine Multimodale Schmerztherapie. Hierbei handelt es sich nicht um das bloße Zusammenstellen einzelner Therapieformen, sondern um ein Konzept, bei dem verschiedene somatische, körperlich und psychologisch übende Verfahren nach vorgegebenem Behandlungsplan und mit identischem Therapieziel anwendet werden [5]. Eine solche Schmerztherapie wird, anders als z. B. häufig in einer Rehabilitationsmaßnahme, in einer festen Patientengruppe durchgeführt. So kann genug Ruhe und Vertrauen entstehen, um den ganzen Menschen, also alle biologischen, psychischen und soziokulturellen Faktoren, die zur Entstehung und Aufrechterhaltung der chronischen Schmerzen beitragen, zu berücksichtigen.

Merke: Die Wirkung der Multimodalen Schmerztherapie ist bei beiden Geschlechtern sehr gut belegt. Allerdings scheinen Frauen noch besser als Männer zu profitieren [20]. Eventuell stehen Frauen den Programminhalten offener gegenüber, sind geübter in Körpertherapieverfahren oder haben einen größeren Nutzen von der Kommunikation in einer Gruppe als Männer.

\section{Arzt oder Ärztin - Patient oder Patientin}

Auch bei der Entscheidung für eine Diagnose oder eine Therapie spielt das Geschlecht von Arzt und Patient eine Rolle. Die höhere Bereitschaft von Frauen, ärztliche Hilfe zu suchen oder Medikamente einzunehmen, wird immer wieder beschrieben.

Zumindest bei der Untersuchung von 2 der häufigsten Schmerzerkrankungen, Rückenschmerzen und Kopfschmerzen, konnten keine eindeutigen Belege für diese Hypothese gefunden werden [21].

\section{Patient oder Patientin}

Allerdings konnte in einer Studie gezeigt werden, dass Frauen anders anamnestisiert werden als Männer. Ihnen werden z.B. eher
Fragen zu psychosozialen Belastungen gestellt als Männern [22]. Männern wird oft unterstellt, ihren Körper als gut oder schlecht funktionierende Maschine wahrzunehmen. Dazu passend fand diese Untersuchung, dass Ärzte bei Frauen mit Beschwerden an der Halswirbelsäule eher zu unspezifischen Diagnosen tendierten, als bei Männern mit dem gleichen Problem [22].

Dieses Phänomen hat aber auch strukturelle Ursachen. Die ICD10-Kriterien einer depressiven Störung (z. B. Antriebslosigkeit, Mangel an Interesse, gedrückte Stimmung, Beeinträchtigung des Selbstwertgefühls) finden sich eher bei erkrankten Frauen. Männer mit Depressionen tendieren häufiger zu Alkoholabusus oder gesteigerter Aggressivität, also zu externalisierenden Verhaltensweisen und werden deshalb eventuell später diagnostiziert als Frauen [23].

\section{OP-Indikationen}

Möglicherweise werden auch Operationsindikationen bei Männern und Frauen unterschiedlich gestellt. Eine schwedische Untersuchung, die sich auf das Swedish Spine Register bezieht, berichtet z. B., dass mehr männliche als weibliche Patienten wegen eines lumbalen Bandscheibenvorfalls operiert wurden. Die Autoren stellen allerdings zur Diskussion, ob dies an der möglicherweise unterschied- 
lichen Bereitschaft von Männern und Frauen, sich einer Operation zu unterziehen liegt, oder ob der Eingriff unterschiedlich häufig von den Ärzten empfohlen wurde [24].

\section{Arzt oder Ärztin}

Auch die Tatsache, ob ein Arzt oder eine Ärztin behandelt, kann auf vielfältige Weise Untersuchungsergebnisse und Therapiepläne beeinflussen. Es liegt nahe, solche Tendenzen eher in Gesellschaften mit starren Rollenverhältnissen zu vermuten. Schweden hingegen gilt als Land mit einer vergleichsweise hohen Sensibilität für die Gleichheit der Geschlechter. Umso interessanter ist deshalb, dass eine schwedische Studie deutliche Unterschiede bei Anamnese und Diagnose gezeigt hat, je nachdem, ob ein Arzt oder eine Ärztin beteiligt war [22].

Es wird wiederholt berichtet, dass Ärztinnen empathischer und patientenorientierter kommunizieren als Ärzte. Ärztinnen wird attestiert, sich mehr um partnerschaftliche Arzt-Patienten-Beziehungen zu bemühen und psychosozialen Faktoren in den Gesprächen mehr Raum einzuräumen. Sie scheinen aktiver zuzuhören und Gefühle bei sich und den Patienten eher zuzulassen [25, 26]. Eine vielbeachtete Studie wies nach, dass die Qualität der Versorgung von Patientinnen und Patienten mit Typ-2-Diabetes durch Ärztinnen besser als durch Ärzte war [27]. Geschlechtsspezifische Aspekte in der Arzt-Patienten-Beziehung bei Schmerzkranken wurden hingegen bisher nicht untersucht.

\section{Schmerzschwelle}

Allerdings gibt es Berichte zur Abhängigkeit der Schmerzschwellen vom Geschlecht des Untersuchers. Männliche Probanden scheinen bei weiblichen Untersuchern höhere Schmerzschwellen zu haben [28]. Einer aktuellen klinischen Untersuchung zufolge, ist dies nur bei sehr leichten Schmerzen der Fall. Starke Schmerzen werden weiblichem Personal gegenüber höher angegeben - und zwar von männlichen und weiblichen Patienten [29].

\section{Konsequenzen für die Praxis}

Nihil nocere und Prävention sind immer Basis ärztlichen Handelns. Um nicht zu schaden, ist die Abwägung des Für und Wider einer Therapie entscheidend.

\section{Höheres Risiko für unerwünschte Arzneimittelwirkungen bei Frauen}

Frauen haben ein höheres Risiko für unerwünschte Arzneimittelwirkungen. Für die Schmerztherapie sind Übelkeit nach Opioiden, allergische Reaktionen auf NSAR oder auch das Risiko einer QT-ZeitVerlängerung durch viele in der Schmerztherapie gebräuchliche Substanzen (Amitryptilin, Methadon etc.) zu nennen [18, 30].

$\mathrm{Zu}$ beachten ist auch, dass Frauen und Männer bestimmte Nebenwirkungen wie z.B. Gewichtszunahme oder Potenz- und Libidostörungen eventuell unterschiedlich bewerten.

Risikofaktor weibliches Geschlecht für Chronifizierungsvorgänge Geht es um Prävention, gilt es v.a. Chronifizierungsvorgängen vorzubeugen.

Das weibliche Geschlecht gilt als Risikofaktor für die Entwicklung von chronischen Schmerzen nach Operationen und für die Entwicklung von chronischen Rückenschmerzen.
Für Letztere wurden u. a. folgende Risikofaktoren identifiziert: - katastrophisierende Gedanken,

- Somatisierung,

- Depression,

- körperliche Extrembelastung am Arbeitsplatz,

- niedrige Bildung,

- niedriges Einkommen,

- Rauchen,

- frühere Schmerzen und

- Übergewicht [5].

Viele dieser Einflussgrößen betreffen Frauen häufiger als Männer. Insofern scheint es bei Frauen noch wichtiger als bei Männern zu sein, auf diese Warnzeichen zu achten, um das Risiko einer Chronifizierung frühzeitig zu erkennen und gegensteuern zu können. Die verschiedenen psychosozialen Risikofaktoren (s. Kasten S.13) müssen aber selbstverständlich im Umgang mit beiden Geschlechtern präsent sein. Sensibilität für Geschlechterunterschiede und für die eigene Rolle im Arzt-Patienten-Verhältnis sind hier sehr hilfreich.

Männer sollten mehr unterstützt werden, sich multimodalen Schmerztherapien zu öffnen und komplementäre Therapieverfahren zu nutzen, um genauso gut wie Frauen von diesen Maßnahmen profitieren zu können.

\section{Fazit}

Für eine exakte Diagnose und eine erfolgreiche Behandlung von Männern und Frauen ist es wichtig, biologisch begründete Unterschiede zwischen den Geschlechtern zu kennen und die Auswirkungen dieser Unterschiede auf Krankheitsentstehung und Therapiewirkung oder -nebenwirkung zu beachten.

Besonders bei komplexen Zuständen wie Schmerzen reicht das Wissen über die „Hardware“ allerdings nicht aus. Psychische und soziale Faktoren sind gleichwertig zu berücksichtigen und in die Therapie mit einzubeziehen. Darüber hinaus müssen wir uns vergegenwärtigen, dass nicht nur Patientinnen und Patienten ihre eigenen Lebenserfahrungen, Rollen und Überzeugungen in die Arzt-Patienten-Beziehung einbringen, sondern auch wir Ärztinnen und Ärzte.

Für die Zukunft benötigen wir also noch mehr als bisher Forschung, die sich mit den unterschiedlichen psychischen, sozialen und kulturellen Einflüssen auf Schmerzen bei Frauen und Männern beschäftigt. Darüber hinaus ist von großer Bedeutung, der Forderung des Gesetzgebers nachzukommen und die Wirksamkeit und Sicherheit von Medikamenten bei beiden Geschlechtern zu belegen.

Eine große Aufgabe wird darin bestehen, geschlechtsspezifische Unterschiede bei der Funktion unseres Gehirns v. a. in Bezug auf Interaktionen - Stichwort Arzt-Patienten-Beziehung - besser zu verstehen.

Interessenkonflikt: Die Autorin erklärt, dass keine wirtschaftlichen oder persönlichen Verbindungen bestehen.

Online zu finden unter

http://dx.doi.org/10.1055/s-0034-1381967

\footnotetext{
Literatur

1 Bonica JJ. The need of a taxonomy. Pain 1979; 6 (3): 247-248

2 van Hecke O, Torrance N, Smith BH. Chronic pain epidemiology and its clinical relevance. $\mathrm{Br}$ J Anaesth 2013; 111 (1): 13-18
} 
$\overline{3}$ Greenspan JD, Craft RM, LeResche L, et al. Studying sex and gender differences in pain and analgesia: a consensus report. Pain 2007; 132 (Suppl 1): S26-45

$\overline{4}$ Ruau D, Liu LY, Clark JD et al. Sex differences in reported pain across 11,000 patients captured in electronic medical records. J Pain 2012; 13 (3): 228-234

5 Arnold B, Brinkschmidt T, Casser HR, et al. Multimodale Schmerztherapie. Schmerz 2009; 23 (2): 112-120

$\overline{6}$ Schnabel A, Pogatzki-Zahn E. Prädiktoren für chronische Schmerzen nach Operationen. Schmerz 2010; 24: 517-533

7 Schopper M, Fleckenstein J, Irnich D. Geschlechtsspezifische Aspekte bei akuten und chronischen Schmerzen. Schmerz 2013; 27 (5): 456-466

$\overline{8}$ Racine M, Tousignant-Laflamme Y, Kloda LA, et al. A systematic literature review of 10 years of research on sex/gender and experimental pain perception - part 1: are there really differences between women and men? Pain 2012; 153 (3): 602-618

$\overline{9}$ Young E, Korszun A. Sex, trauma, stress hormones and depression. Mol Psychiatry 2010; 15 (1): 23-28

$\overline{10}$ Nelson S, Baldwin N, Taylor J. Mental health problems and medically unex-plained physical symptoms in adult survivors of childhood sexual abuse: an integrative literature review. J Psychiatr Ment Health Nurs 2012; 19 (3): 211-220

$\overline{11}$ Finkelhor D, Hotaling G, Lewis IA, et al. Sexual abuse in a national survey of adult men and women: prevalence, characteristics, and risk factors. Child abuse \& neglect 1990; 14 (1): 19-28

$\overline{12}$ Keogh E, Eccleston C. Sex differences in adolescent chronic pain and pain-related coping. Pain 2006; 123 (3): 275-284

$\overline{13}$ Teuber N, Thiele A, Eberhardt B. Geschlechtsrolle und Schmerzerleben. Schmerz 2006; 20 (4): 307-313

$\overline{14}$ Bundesgesetzblatt. Zwölftes Gesetz zur Änderung des Arzneimittelgesetzes. www.bgbl.de: Bundesanzeiger Verlagsgesellschaft mbH; 2004

$\overline{15}$ Xie HG, Kim RB, Wood AJ, et al. Molecular basis of ethnic differences in drug disposition and response. Annu Rev Pharmacol Toxicol 2001; 41: 815-850

$\overline{16}$ Craft RM. Sex differences in drug- and non-drug-induced analgesia. Life sciences 2003; 72 (24): 2675-2688

$\overline{17}$ Smith YR, Stohler CS, Nichols TE, et al. Pronociceptive and antinociceptive effects of estradiol through endogenous opioid neurotransmission in women. J Neurosci 2006; 26 (21): 5777-5785

$\overline{18}$ Rademaker M. Do women have more adverse drug reactions? Am J Clin Dermatol 2001; 2 (6): 349-351

$\overline{19}$ Witt CM, Schutzler L, Ludtke R, et al. Patient characteristics and variation in treatment outcomes: which patients benefit most from acupuncture for chronic pain? Clin J Pain 2011; 27 (6): 550-555

$\overline{20}$ Pieh C, Altmeppen J, Neumeier S, et al. Gender differences in outcomes of a multimodal pain management program. Pain 2012; 153 (1): 197-202

$\overline{21}$ Hunt K, Adamson J, Hewitt C, et al. Do women consult more than men? A review of gender and consultation for back pain and headache. J Health Serv Res Policy 2011; 16 (2): 108-117

$\overline{22}$ Hamberg K, Risberg G, Johansson EE, et al. Gender bias in physicians' management of neck pain: a study of the answers in a Swedish national examination. Journal of women's health \& gender-based medicine 2002; 11 (7): 653-666

$\overline{23}$ Möller-Leimkühler AMP, N.C.; Heller J. Male Depression. der Mann - wissenschaftliches Journal für Männergesundheit 2009; 7 (4): 15-20

$\overline{24}$ Stromqvist B, Fritzell P, Hagg O, et al. The Swedish Spine Register: development, design and utility. Eur Spine J 2009; 18 (Suppl 3): S294-304

$\overline{25}$ Roter DL, Hall JA, Aoki Y. Physician gender effects in medical communication: a meta-analytic review. JAMA 2002; 288 (6): 756-764

$\overline{26}$ Arnold RM, Martin SC, Parker RM. Taking care of patients - does it matter whether the physician is a woman? The Western journal of medicine 1988; 149 (6): 729-733 $\overline{27}$ Berthold HK, Gouni-Berthold I, Bestehorn KP, et al. Physician gender is associated with the quality of type 2 diabetes care. Journal of internal medicine 2008; 264 (4): 340-350

$\overline{28}$ Levine FM, De Simone LL. The effects of experimenter gender on pain report in male and female subjects. Pain 1991; 44 (1): 69-72

$\overline{29}$ Vigil JM, Alcock J. Tough guys or sensitive guys? Disentangling the role of examiner sex on patient pain reports. Pain research \& management: the journal of the Canadian Pain Society = journal de la societe canadienne pour le traitement de la douleur 2014; 19 (1): e9-e12

$\overline{30}$ Alvarez PA, Pahissa J. QT alterations in psychopharmacology: proven candidates and suspects. Current drug safety 2010; 5 (1): 97-104

$\overline{31}$ Hasenbring M PM. Psychologische Mechanismen der Chronifizierung - Konsequenzen für die Prävention. In: Basler HD FC, Kröner-Herwig B, Rehfisch HP, Hrsg. Psychologische Schmerztherapie. 5. Aufl. Berlin: Springer; 2004: 99-118

$\overline{32}$ Kendall NaS, Linton S], Main CJ. Guide to Assessing Psychosocial Yellow Flags in Acute Low Back Pain: Risk Factors for Long-Term Disability and Work Loss. In: Disability NACoHa (ed) Accident Rehabilitation \& Compensation Insurance Corporation of New Zealand, and the National Health Committee, Ministry of Health. New Zealand: Wellington; 1997

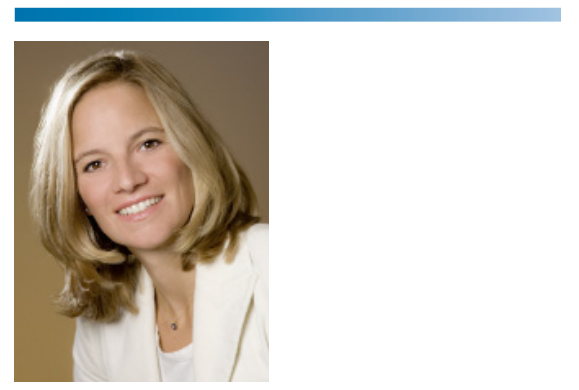

Dr. med. Miriam Schopper

Interdisziplinäre Schmerzambulanz -

Campus Innenstadt

Klinik für Anästhesiologie

Klinikum der Universität München

Pettenkoferstr. $8 a$

80336 München

miriam.schopper@med.uni-muenchen.de

Miriam Schopper ist Fachärztin für Anästhesiologie. Nach Station in der Kardioanästhesie war sie mehrere Jahre in der interdisziplinären Schmerzambulanz der LMU tätig; während dieser Zeit u. a. Leitung eines integrierten Versorgungsprojekts in der Tagesklinik für chronisch Schmerzkranke. Weitere Schwerpunkte waren die Kombination von komplementären (Akupunktur) und schulmedizinischen Verfahren bei chronischen Schmerzpatienten. Als Anästhesistin und Schmerztherapeutin folgte ein Jahr in der Klinik für Palliativmedizin.

Durch die vielen Jahre interdisziplinärer Tätigkeit entstand ihr Interesse an gendermedizinschen Fragestellungen. Zu diesem Thema erhält sie international Vortragseinladungen. Seit 2011 leitet sie die interdisziplinäre Lehrveranstaltung „Gendermedizin“ an der Ludwig-Maximilian-Universität München. 